Pacific Journal of Mathematics

TRACE RINGS FOR VERBALLY PRIME ALGEBRAS 


\title{
TRACE RINGS FOR VERBALLY PRIME ALGEBRAS
}

\section{Allan Berele}

\begin{abstract}
Every algebra p.i. equivalent to some $M_{k, l}$ and with zero annihilator of the Razmyslov ideal has a central extension with non-degenerate trace.
\end{abstract}

A p.i. algebra $R$ is said to be verbally prime if whenever $f\left(x_{1}, \ldots, x_{n}\right) g\left(x_{n+1}, \ldots, x_{m}\right)$ is an identity for $R$ then either $f$ or $g$ is. Kemer introduced these algebras in [3]. Since then, verbally prime algebras have been the subject of a number of papers. At the end of this paper we will summarize the results known to us. A common theme is that verbally prime algebras have many properties in common with prime p.i. algebras. In the present work we continue this thread and show that certain verbally prime algebras have "nice" embeddings into trace rings.

Kemer proved that every non-trivial verbally prime p.i. algebra in characteristic zero must be p.i. equivalent to either: $n \times n$ matrices over the field; or $n \times n$ matrices over an infinite dimensional Grassmann algebra $E$; or $M_{k, l}$. The algebra $M_{k, l}$ is a certain subalgebra of the $(k+l) \times(k+l)$-matrices over $E . E$ has a natural $Z / 2 Z$-grading $E=E_{0} \oplus E_{1}$, in which $E_{0}$ is spanned by the even words and $E_{1}$ is spanned by the odd words. Then $M_{k, l}$ consists of all $(k+l) \times(k+l)$ matrices of the form $\left(\begin{array}{ll}A & B \\ C & D\end{array}\right)$, where $A$ is a $k \times k$-matrix with entries in $E_{0}, D$ is an $l \times l$-matrix with entries $E_{0}$, and $B$ and $C$ have entries in $E_{1}$.

The algebra $M_{k, l}$ has a trace function $\operatorname{tr}: M_{k, l} \rightarrow E_{0}$ defined by $\operatorname{tr}\left(\left(\begin{array}{ll}A & B \\ C & D\end{array}\right)\right)=\operatorname{tr} A-\operatorname{tr} D$. This function satisfies all of the usual properties of the usual properties of trace: it takes values in $E_{0}$, the center of $M_{k, l}$; it is $E_{0}$-linear; and $\operatorname{tr}(x y)=\operatorname{tr}(y x)$ for all $x, y \in M_{k, l}$. Razmyslov [5] studied the central polynomials and trace identities of $M_{k, l}$. He found non-vanishing, multilinear central polynomials $p\left(x_{1}, \ldots, x_{n}, a\right)$ and $c\left(x_{1}, \ldots, x_{n}\right)$ for $M_{k, l}$ with the property that

$$
p\left(x_{1}, \ldots, x_{n}, a\right)=c\left(x_{1}, \ldots, x_{n}\right) \operatorname{tr}(a)
$$

on $M_{k, l}$. For convenience, we generally abbreviate $p\left(x_{1}, \ldots, x_{n}, a\right)$ to $p(x, a)$ and $c\left(x_{1}, \ldots, x_{n}\right)$ to $c(x)$. 
In this paper we will work with algebras $R$ which are p.i. equivalent to $M_{k, l}$, for some $k, l$. (Our base field need not be characteristic zero. In the characteristic $p$ case $M_{k, l}$ is known to be verbally prime, but Kemer's classification theorem may not hold.) And, we will need one more hypothesis. Let $J=$ the ideal of $R$ generated by all evaluations of $c(x)$. We will call $J$ the Razmyslov ideal of $R$. Our final hypothesis is that $J$ should have no annihilator in $R$. Here is our theorem:

THEOREM. Assume that $R$ is p.i. equivalent to some $M_{k, l}$ and that the annihilator of $J$ is (0). Then there is embedding $R \rightarrow \bar{R}$ of $R$ into a central extension $\bar{R}$ where

(1) $\bar{R}$ has a non-degenerate trace,

(2) for all $r \in \bar{R}$ there is an integer $n$ such that $J^{n} r \subset R$, and

(3) $\bar{R}$ satisfies exactly the same trace identities as $M_{k, l}$.

We start off by using the equation $\left({ }^{*}\right)$ to investigate the properties of $p\left(x_{1}, \ldots, x_{n}, a\right)$ and $c\left(x_{1}, \ldots, x_{n}\right)$. Equation $\left(^{*}\right)$ is not defined in $R$, since $R$ has no trace function. However, any consequence of $\left(^{*}\right)$ which does not involve trace will be a polynomial identity in $R$ since it will be identity in $M_{k, l}$.

One obvious consequence is

$$
p(x, a b)=p(x, b a)
$$

since $\operatorname{tr}(a b)=\operatorname{tr}(b a)$. Also, since

$$
c(x) \operatorname{tr}(a) c(y) \operatorname{tr}(b)=c(y) \operatorname{tr}(a) c(x) \operatorname{tr}(b),
$$

we get

$$
p(x, a) p(y, b)=p(y, a) p(x, b) .
$$

Similarly, since $c(x) c(y) \operatorname{tr}(a)=c(y) c(x) \operatorname{tr}(a)$,

$$
c(x) p(y, a)=c(y) p(x, a) .
$$

We now prove a slightly deeper lemma.

LEMMA. If $\sum_{i} c\left(x_{i}\right) r_{i}=0$ then $\sum_{i} p\left(x_{i}, a\right) r_{i}=0$ for all $a$.

Proof. Choose any evaluation of $c, c(z)$. 
Now calculate

$$
\begin{aligned}
c(z) \sum_{i} p\left(x_{i}, a\right) r_{i} & =\sum_{i} c(z) p\left(x_{i}, a\right) r_{i} \\
& =\sum_{i} c\left(x_{i}\right) p(z, a) r_{i} \quad \text { by }(3) \\
& =p(z, a) \sum_{i} c\left(x_{i}\right) r_{i} \\
& =0
\end{aligned}
$$

Hence, $J \sum_{i} p\left(x_{i}, a\right) r_{i}=0$. But ann $J=(0)$ and so $\sum_{i} p\left(x_{i}, a\right) r_{i}=$ 0 , as claimed.

This allows us to make the following definition:

Definition. Let $a \in R$. We define $\operatorname{tr}(a)$, which will be an element of $\operatorname{Hom}_{R}(J, R)$, the set of $R$-module maps from $J$ to $R$, by

$$
\operatorname{tr}(a)(c(s) r)=p(x, a) r .
$$

The symbol $\operatorname{tr}($ ) satisfies the usual properties of a trace function: It is linear, it vanishes on commutators (by (1)) and it is central, in some sense. We now need to define the trace ring of $R$, which will be generated by $R$ and $\{\operatorname{tr}(a) \mid a \in R\}$. To this end we need the following little lemma:

Lemma. The natural maps $i_{n}: \operatorname{Hom}_{R}\left(J^{n}, R\right) \rightarrow \operatorname{Hom}_{R}\left(J^{n+1}, R\right)$ gotten by restriction are injections. Moreover, the image of $i_{n}$ is contained in $\operatorname{Hom}_{R}\left(J^{n+1}, J\right)$.

Proof. Assume that there was a non-zero map $f: J^{n} \rightarrow R$ which vanished on $J^{n+1}$. Let $I=$ the image of $f$. For all $c(x), c(x) I=$ $c(x) f\left(J^{n}\right)=f\left(c(x) J^{n}\right) \subset f\left(J^{n+1}\right)=0$. Hence $I \subset$ ann $J$ and so $I=0$. So $f$ must be the zero map.

Also note that $f\left(J^{n+1}\right)=J f\left(J^{n}\right) \subset J R$.

Using this lemma we take the direct limit (union) of $\operatorname{Hom}_{R}\left(J^{n}, R\right)$ and call it $A . A$ is an additive group and it contains a copy of $R$ (acting by left multiplication). $A$ can also be given a multiplicative structure, using composition of functions. Given $f: J^{n} \rightarrow R$ and $g: J^{m} \rightarrow R$ we restrict $g$ to a map $J^{m+n} \rightarrow J^{n}$ and define $f \cdot g: J^{m+n} \rightarrow R$ by composition of functions. Then $\bar{R}$ will be the subalgebra of $A$ generated by $R$ and $\{\operatorname{tr}(a) \mid a \in R\}$. If 
$f \in \operatorname{Hom}_{R}\left(J^{n}, R\right)$ is any element of $\bar{R}$, we may define $\operatorname{tr}(f) \in$ $\operatorname{Hom}_{R}\left(J^{n+1}, R\right)$ via $\operatorname{tr}(f)(c(x) r)=p(x, f) r$. It is not too hard to show that $\operatorname{tr}(f)$ will also belong to $\bar{R}$ and that it will be central in $\bar{R}$.

We remark the annihilator of $J$ in $A$ (and hence in $\bar{R}$ ) will still be zero. For, if $f \in \operatorname{Hom}_{R}\left(J^{n}, R\right)$ were such that $J f=0$, then $f\left(J^{n+1}\right)=J f\left(J^{n}\right)=0$ and so $i_{n}(f)$ would be zero.

We also remark that the element $\operatorname{tr}\left(a_{1}\right) \operatorname{tr}\left(a_{2}\right) \cdots \operatorname{tr}\left(a_{n}\right)$ of $\operatorname{Hom}_{R}\left(J^{n}, R\right)$ takes $c\left(x_{1}\right) \cdots c\left(x_{n}\right)$ to $p\left(x_{1}, a_{1}\right) \cdots p\left(x_{n}, a_{n}\right)$ and that this is well-defined by (2). We also remark that our construction of $A$ obviously is based on the construction of the Martingale ring of quotients. Here, now, is our main result:

Theorem. Assume that $R$ is p.i. equivalent to $M_{k, l}$ and that the Razmyslov ideal $J$ has zero annihilator. Then $R$ embeds into a ring $\bar{R}$ with trace, as in the above construction such that

(1) for all $r \in \bar{R}$ there exists an $n$ such that $r J^{n} \subset R$,

(2) $\bar{R}$ satisfies the same trace identities as $M_{k, l}$,

(3) the trace on $\bar{R}$ is non-degenerate.

Proof. (1) If $r \in \bar{R}$, then $r \in \operatorname{Hom}_{R}\left(J^{n}, R\right)$ for some $n$, and so $r J^{n} \subset R$.

(2) Let $T\langle X\rangle$ be the free (associative) algebra with trace on the infinite set $X$. So trace polynomials are elements of $T\langle X\rangle$. Let $F\langle X\rangle \subset T\langle X\rangle$ be the free (associative) algebra on $X$, corresponding to ordinary polynomials. Let $I\left(M_{k, l}\right)$ and $I(\bar{R})$ be the ideals of trace identities for $M_{k, l}$ and $\bar{R}$, respectively, in $T\langle X\rangle$. By hypothesis

$I\left(M_{k, l}\right) \cap F\langle X\rangle=I(\bar{R}) \cap F\langle X\rangle$.

Let $J_{x}$ be the $T$-ideal of $F\langle X\rangle$ generated by the central polynomial $c\left(x_{1}, \ldots, x_{n}\right)$. Since both $M_{k, l}$ and $\bar{R}$ have no non-zero annihilator of their Razmyslov ideals, it follows that

$$
\begin{aligned}
& \text { if } J_{x} a \in I\left(M_{k, l}\right), \quad \text { then } a \in I\left(M_{k, l}\right) \text { and } \\
& \text { if } J_{x} a \in I(\bar{R}), \quad \text { then } a \in I(\bar{R}) .
\end{aligned}
$$

Let $T_{n}\langle X\rangle$ be the space of trace polynomials of total degree $n$. Modulo the relation $\left(^{*}\right) J_{x}^{n} T_{n}\langle X\rangle \subset F\langle X\rangle$. But, each of $I\left(M_{k, l}\right)$ and $I(\bar{R})$ contain $\left(^{*}\right)$. Hence

$$
\begin{aligned}
& J_{x}^{n}\left(I\left(M_{k, l}\right) \cap T_{n}\langle X\rangle\right) \subset I\left(M_{k, l}\right) \cap F\langle X\rangle \bmod I(\bar{R}) \quad \text { and } \\
& J_{x}^{n}\left(I(\bar{R}) \cap T_{n}\langle X\rangle\right) \subset I(\bar{R}) \cap F\langle X\rangle \bmod I\left(M_{k, l}\right) .
\end{aligned}
$$

The proof now easily follows. 
(3) To prove (3) we resort to another identity of Razmyslov. Razmyslov proved that there exist polynomials $e_{i}\left(x_{1}, \ldots, x_{n}\right)$ and $e_{i}^{\prime}\left(x_{1}, \ldots, x_{n}\right)$ such that

$(* *)$

$$
c(x) a-\sum_{i} \operatorname{tr}\left(e_{i}(x) a\right) e_{i}^{\prime}(x)
$$

is a trace identity for $M_{k, l}$. By part (2) (**) will also hold in $\bar{R}$. Now assume that $\operatorname{tr}(a R)=0$ for some $a \in R$. But in (**) this would imply that $c(x) a=0$ for all $c(x)$. Hence $0 \neq a \in$ ann $J=(0)$. More generally, if $\operatorname{tr}(a \bar{R})=0$ for some $0 \neq a \in \bar{R}$, we may choose a $0 \neq b \in R \cap a J^{n}$ for some $n$. But $b R \subset a \bar{R}$, yielding the contradiction $\operatorname{tr}(b R)=0$.

We point out that the trace also behaves well with respect to ideals.

Proposition. Let $I$ be an ideal of $\bar{R}($ or $R)$. Then $J \operatorname{tr}(I) \subset I$.

Proof. If $a \in I$, then $c(x) \operatorname{tr}(a)=p(x, a) \in I$.

Proposition. If $I_{1}, I_{2}$ are ideals of $\bar{R}$, then $I_{1} I_{2}=(0)$ if and only if $\operatorname{tr}\left(I_{1}\right) \operatorname{tr}\left(I_{2}\right)=(0)$.

Proof. If $I_{1} I_{2}=0$ then, by the above $J^{2} \operatorname{tr}\left(I_{1}\right) \operatorname{tr}\left(I_{2}\right)=(0)$.

If $\operatorname{tr}\left(I_{1}\right) \operatorname{tr}\left(I_{2}\right)=(0)$, choose any $c(x)$ and $c(y)$ and apply $\left(^{* *}\right)$.

$$
\begin{aligned}
c(x) c(y) I_{1} I_{2} & =\left(c(x) I_{1}\right)\left(c(y) I_{2}\right) \\
& =\sum_{i} \operatorname{tr}\left(e_{i}(x) I_{1}\right) e_{i}^{\prime} \sum_{j} \operatorname{tr}\left(e_{j}(y) I_{2}\right) e_{j}^{\prime} \\
& \subset \sum_{i, j} \operatorname{tr}\left(I_{1}\right) \operatorname{tr}\left(I_{2}\right) e_{i}^{\prime} e_{j}^{\prime}=(0) .
\end{aligned}
$$

COROLlary. $I^{n}=(0)$ if and only if $\operatorname{tr}(I)^{n}=(0)$.

We also point out that this trace behaves well with respect to homomorphisms.

Proposition. Let $\varphi: R \rightarrow S$ be an algebra homomorphism and assume that

(1) $R$ is p.i. equivalent to $M_{k, l}$ and no non-zero element of $R$ annihilates the Razmyslov ideal, $J$,

(2) $S$ has a trace function and $S$ is trace-p.i. equivalent to $M_{k, l}$, and

(3) the annihilator of $\varphi(J)$ in $S$ is (0).

Then $\varphi$ extends to a trace preserving homomorphism from $\bar{R}$ to $S$. 
Proof. We want to define $\bar{\varphi}: \bar{R} \rightarrow S$ via $\bar{\varphi}(\operatorname{tr}(a))=\operatorname{tr}(\varphi(a))$. The only problem is showing that this $\bar{\varphi}$ is well-defined.

Let $f\left(x_{1}, \ldots, x_{n}\right)$ be any (formal) trace polynomial such that $f\left(a_{1}, \ldots, a_{n}\right)=0$ for some choice of $a_{1}, \ldots, a_{n} \in R$. We need to prove that $f\left(\varphi\left(a_{1}\right), \ldots, \varphi\left(a_{n}\right)\right)=(0)$ in $S$.

By $\left(^{*}\right)$ there exists an integer $k$ and an ordinary (not trace) polynomial $g$ such that $c\left(y_{1}\right) c\left(y_{2}\right) \cdots c\left(y_{k}\right) f(x)-g(x, y)$ is the identity for $M_{k, l}$. This implies, on the one hand, that $g(a, y)=0$ in $R$ for all choices of $y$ and, on the other hand, that $c\left(\varphi\left(y_{1}\right)\right) \cdots c\left(\varphi\left(y_{k}\right)\right) f(\varphi(a))$ $=0$ in $S$. Hence, $f\left(\varphi\left(a_{1}\right), \ldots, \varphi\left(a_{k}\right)\right) \in \operatorname{Ann}\left(\varphi\left(J^{k}\right)\right)$ and so $f(\varphi(a))$ $=0$ as claimed.

To see the necessity of the hypothesis ann $J=(0)$, let $E$ be the Grassmann algebra (with unit) on the infinite dimensional $F$-vector space $V$, and let $Z$ be the center of $E$. Then our algebra will be $R=$ $E \otimes_{Z} E$. Kemer proved that $E \otimes E$ is p.i. equivalent to $M_{1,1}$. We do not know if there is an embedding $R$ into $\bar{R}$, where $\bar{R}$ has a nondegenerate trace and is trace p.i. equivalent to $M_{1,1}$. However, if such an embedding of $R$ did exist it would not have the good properties of the one we constructed. Specifically, assume that $i: E \otimes E \rightarrow R$ is an embedding, that $R$ has a non-degenerate $Z(R)$ - and $Z(E \otimes E)$-linear trace function and that $R$ is trace p.i. equivalent to $M_{1,1}$. Then

(1) the restriction of tr to $E \otimes E$ is not non-degenerate,

(2) $R$ is not a central extension of $E \otimes E$, and

(3) for some $r \in R$ there does not exist $z \in Z(E \otimes E)$ such that $0 \neq z x \in E \otimes E$. For the proof, note that $\operatorname{tr}(1)=0$ in $M_{1,1}$, hence in $R$. Now, let $0 \neq v \in V$ and let $a=v \otimes v \in E \otimes E$. We leave it as an exercise for the reader to show that if $\operatorname{tr}(a x)=0$, then $x$ does not lie in the ring generated by $Z(R)$ and $E \otimes E$; and that $Z(E \otimes E) \operatorname{tr}(a x)$ does not contain any non-zero elements of $E \otimes E$.

As promised earlier, we conclude with a summary of the theory of verbally prime p.i. algebras. These algebras were introduced by Kemer in [3]. In that work he proved the classification theorem mentioned above. He also proved that any p.i. algebra has a nilpotent ideal modulo which it is equivalent to a direct sum of verbally prime algebras. He also studied the tensor products of verbally prime algebras. He showed that such a product will be verbally prime and gave explicit computational formulas (see [4]). This work has not appeared in the West, but the results have been duplicated by Regev in [6] and [7]. Razmyslov studied verbally prime p.i.'s in [5]. He showed that they 
all admit central polynomials. He also studied the trace identities of $M_{k, l}$ and found that all trace identities for $M_{k, l}$ are consequences of the identities of degree $(k+l) \times(k+l)$. This latter is an analogue of the Razmyslov-Procesi theorem. In [1] we reprove Razmyslov's result using methods which generalize Procesi's use of classical invariant theory. Finally, in [2], we used Razmyslov's central polynomials to get a partial generalization of the Artin-Procesi theorem. We proved that every verbally prime p.i. algebra satisfies properties similar to Azumaya algebras.

We are happy to thank A. Braun for suggesting that we use central polynomials to define traces. We also thank MSRI where we were a guest for the noncommutative ring theory microprogram at which we did part of this work.

\section{REFERENCES}

[1] A. Berele, Trace identities and Z/2Z-graded invariants, Trans. Amer. Math. Soc., 309 (2) (1988), 581-589.

[2] _ Azumaya-like properties of verbally prime algebras, J. Algebra.

[3] A. R. Kemer, Varieties and $Z_{2}$-graded algebras, Math. USSR-Izv., 25 (1985), 359-374.

[4] T. V. Gateva, On the pi-degree of the tensor product of pi-algebras, C.R. Acad. Bulgare Sci., 36 (11) (1983), 1367-1370.

[5] Yu. P. Razmyslov, Trace identities and central polynomials in the matrix superalgebra $M_{k, l}$, Math. USSR-Sb., 56 (1) (1987), 187-206.

[6] A. Regev, Homomorphisms for tensor products of Grassmann algebras, in Ring Theory 1989 in honor of S. A. Amitsor, L. Rowen, editor, The Weizmann Science Press of Israel, Rehovot, Israel, 1989.

[7] _ Tensor products of matrix algebras over the Grassmann algebra, J. Algebra, to appear.

Received January 15, 1990.

De Paul UNiversity

Chicago, IL 60614 



\section{PACIFIC JOURNAL OF MATHEMATICS EDITORS}

\author{
V. S. VARADARAJAN \\ (Managing Editor) \\ -University of California \\ Los Angeles, CA 90024-1555-05 \\ Herbert Clemens \\ University of Utah \\ Salt Lake City, UT 84112 \\ THOMAS ENRIGHT \\ University of California, San Diego \\ La Jolla, CA 92093
}

R. FINN

Stanford University

Stanford, CA 94305

Hermann FlaschKa

University of Arizona

Tucson, AZ 85721

VAUGHAN F. R. JONES

University of California

Berkeley, CA 94720

SteVen KerCKhoff

Stanford University

Stanford, CA 94305
C. C. MOORE

University of California

Berkeley, CA 94720

Martin ScharlemanN

University of California

Santa Barbara, CA 93106

\section{HAROLD STARK}

University of California, San Diego

La Jolla, CA 92093

\section{ASSOCIATE EDITORS}

\begin{tabular}{|c|c|c|c|c|}
\hline R. Arens & $\begin{array}{l}\text { E. F. BECKENBACH } \\
(1906-1982)\end{array}$ & Neumann & $\begin{array}{l}\text { F. WolF } \\
(1904-1989)\end{array}$ & K. Yoshida \\
\hline \multicolumn{5}{|c|}{ SUPPORTING INSTITUTIONS } \\
\hline \multicolumn{2}{|c|}{ UNIVERSITY OF ARIZONA } & \multicolumn{3}{|c|}{ UNIVERSITY OF OREGON } \\
\hline \multirow{2}{*}{\multicolumn{2}{|c|}{$\begin{array}{l}\text { UNIVERSITY OF BRITISH COLUMBIA } \\
\text { CALIFORNIA INSTITUTE OF TECHNOLOGY }\end{array}$}} & \multicolumn{3}{|c|}{ UNIVERSITY OF SOUTHERN CALIFORNIA } \\
\hline & & \multicolumn{3}{|c|}{ STANFORD UNIVERSITY } \\
\hline CALIFORNIA INSTITUTE OF TECHNOLOGY & UNIVERSITY OF CALIFORNIA & \multicolumn{3}{|c|}{ UNIVERSITY OF HAWAII } \\
\hline \multicolumn{2}{|c|}{ MONTANA STATE UNIVERSITY } & \multicolumn{3}{|c|}{ UNIVERSITY OF TOKYO } \\
\hline \multicolumn{2}{|c|}{ UNIVERSITY OF NEVADA, RENO } & \multicolumn{3}{|c|}{ UNIVERSITY OF UTAH } \\
\hline \multicolumn{2}{|c|}{ NEW MEXICO STATE UNIVERSITY } & \multicolumn{3}{|c|}{ WASHINGTON STATE UNIVERSITY } \\
\hline OREGON STA & UNIVERSITY & UNIVERS & OF WASHING & \\
\hline
\end{tabular}




\section{Pacific Journal of Mathematics}

Vol. 150, No. $1 \quad$ September, 1991

Jan Aarts, Charles Lemuel Hagopian and Lex Gerard Oversteegen, The orientability of matchbox manifolds $\ldots \ldots \ldots \ldots \ldots \ldots \ldots \ldots \ldots \ldots \ldots \ldots$

Roger W. Barnard, Carl Hanson Fitzgerald and Sheng Gong, The growth and $1 / 4$-theorems for starlike mappings in $\mathbf{C}^{n} \ldots \ldots \ldots \ldots \ldots \ldots \ldots$

Allan Berele, Trace rings for verbally prime algebras $\ldots \ldots \ldots \ldots \ldots \ldots \ldots 23$

Quo-Shin Chi, Curvature characterization and classification of rank-one

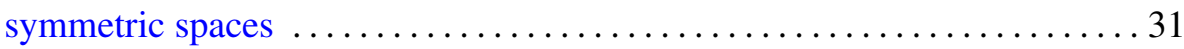

Harold Gerard Donnelly and Jeffrey Marc Lee, Domains in Riemannian

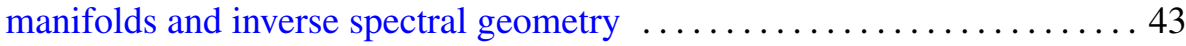

Clifford John Earle, Jr. and Patricia Lilaine Sipe, Families of Riemann

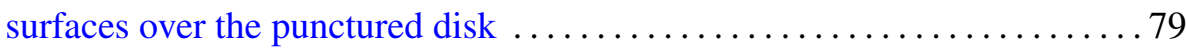

Mark P. Hughes, Dihedral group actions on homotopy complex projective

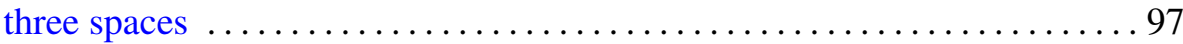

Robert F. Lax and Carl Widland, Gap sequences at a singularity ........111

Takahiko Nakazi, Bounded Hankel forms with weighted norms and lifting

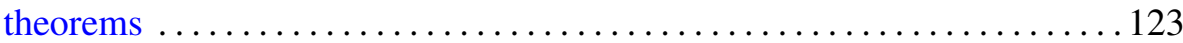

Douglas Murray Pickrell, Mackey analysis of infinite classical motion groups

Martha Rzedowski-Calderón and Gabriel Daniel Villa-Salvador,

Automorphisms of congruence function fields 167

Peter N-S Wong, Equivariant Nielsen fixed point theory for $G$-maps 